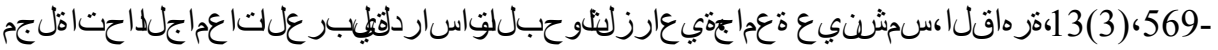
579، 2005

]38[

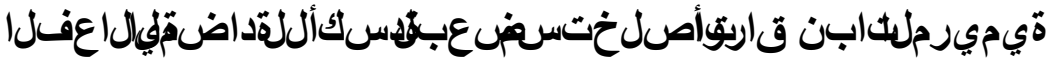

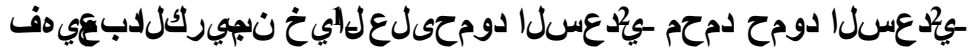

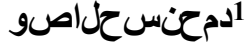

\section{ز ز-}

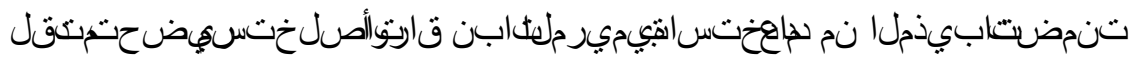

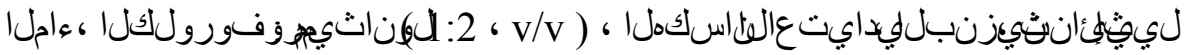

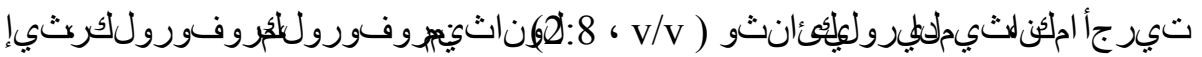

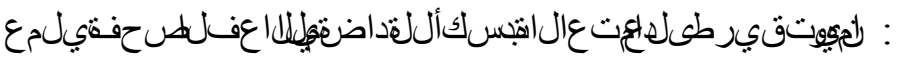

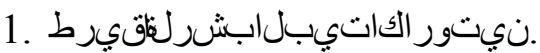

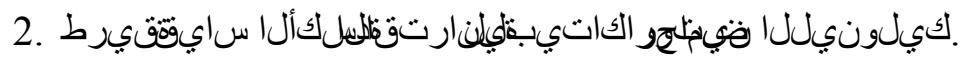

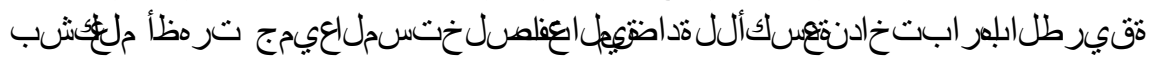

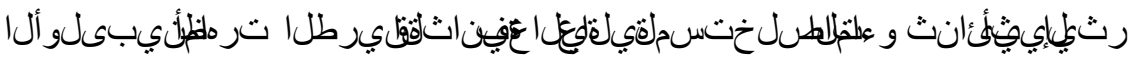

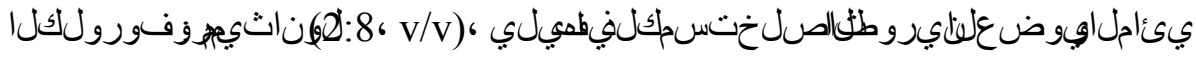

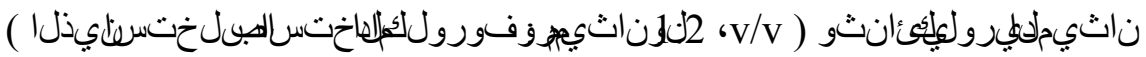

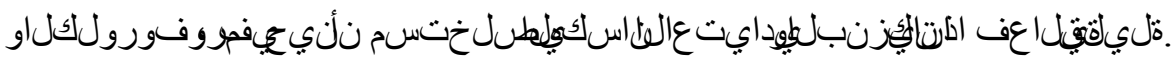

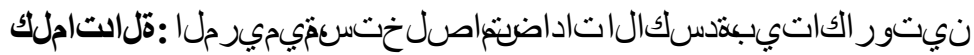

$$
\text { قـمدقىم }
$$

Sيlvia officinalis L.) ئيديرملثكابن

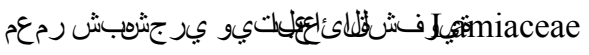

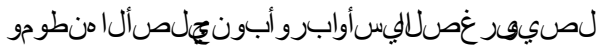

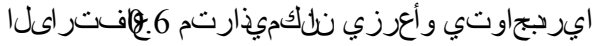

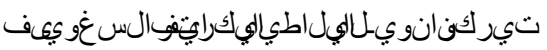

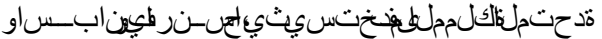

$$
\begin{aligned}
& \text { ايونس نط } 2 \text { و نط 30ود حببتيز كوقةفاج قارو أك }
\end{aligned}
$$

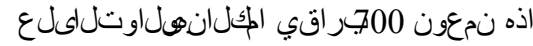

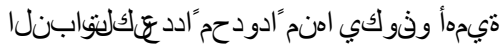
.

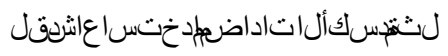

(Propyl gallate) PG و (Butylated BHT, hydroxy toluene)

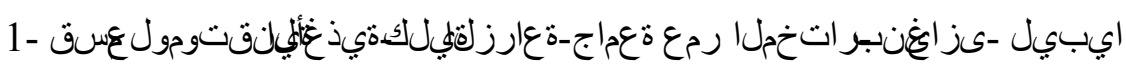

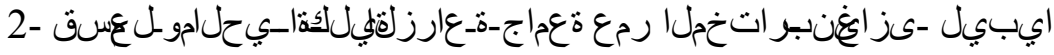


569

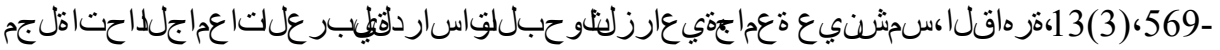
579، 2005

(Butylated BHA), hydroxyl anisol) و

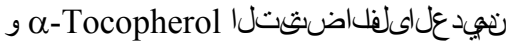

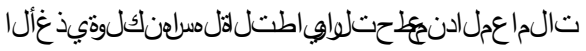

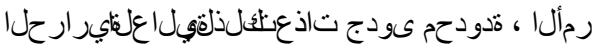

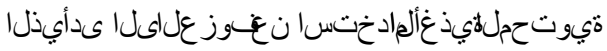

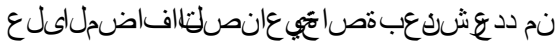

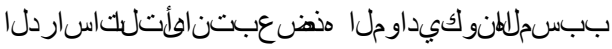

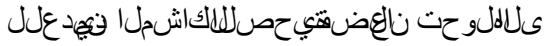
(Johnson \&

Hewgill, 1961 and Branen, 1975)

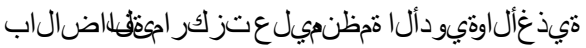

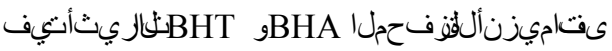

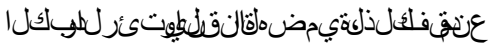

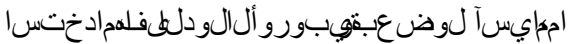

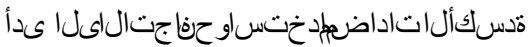

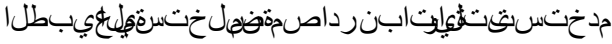

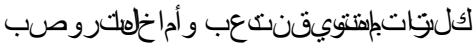

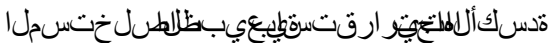

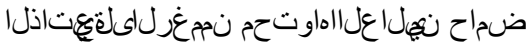

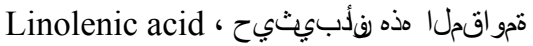

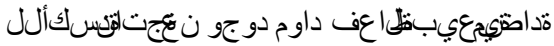

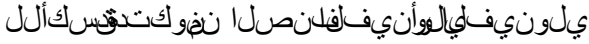
(Bracco et al 1981) .

ةداضثلهاعفتابكرم دو جوىلريشأدقو لب جلبليلكئل

(Chang et al 1977 and Svoboda, 1992)

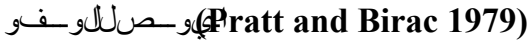
Wu et al
1982; Economou et al 1991 and

Cuvelier et al 1994) (Miura and لفلفلاو (1989) لفنفلاو (\$akatani et al 1986)

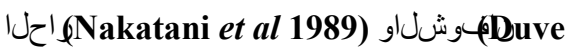
and White, 1991) (Jitoe et al 1992 and Kikuzaki \& Nakatani, 1993)

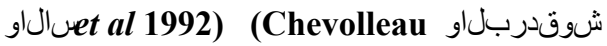
Vekiari et al (1993) زلعاوشقو)(Eisa, 1999).

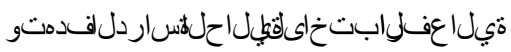

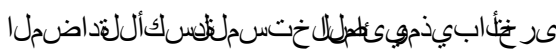

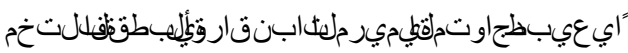
رضخ أللاب جلةقطن

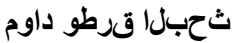

قيت تابنقهي عل

تاحش )يحاونثالث ثنيجيرملتابن عمجتت لبجلةقطنم نله اتخملا رمعوفوكل اكل يداوو

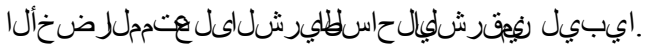

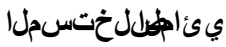

$$
\text { Miller }
$$
نم مج 50 ذخلمتثي

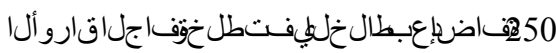

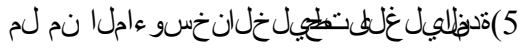

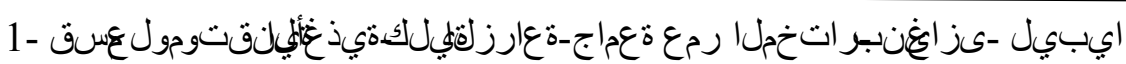

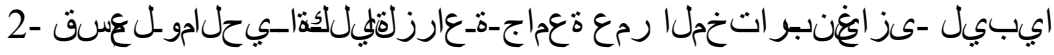




$$
\text { ثيجيرتملطسلختسمبتدسكأل }
$$

كن خاسلاءاحل نم لم 200بلس غوحشرمثنقئاقد

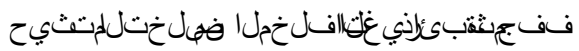

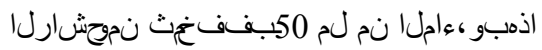

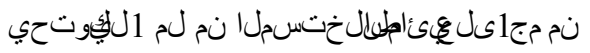
تب تمئاذلاداوملا.

ي ئ املا يليوضعلنهيروطثاصلختسم

بئيصلختسمجلاصفنيلمعتيرجأ

ط Folch and Sloanestanley (1957) مروفورولكلابيذم نم لم 500بيضك لثيح

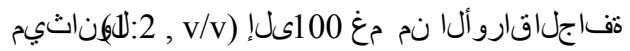

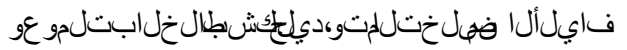

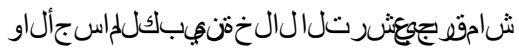

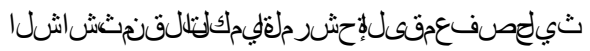

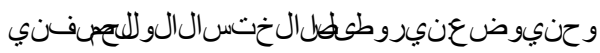

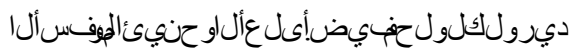

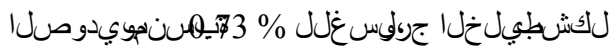

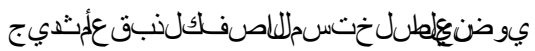
امززيكرتثث نمومقلا نم ةدحىل علكئئاملاو

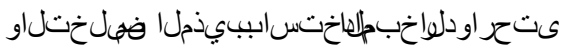

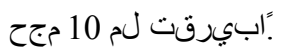

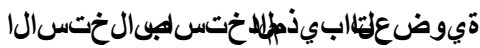

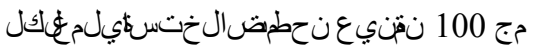

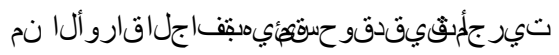

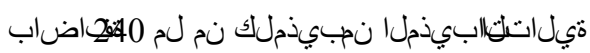

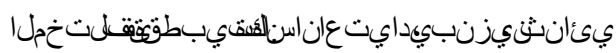

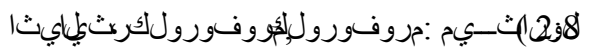

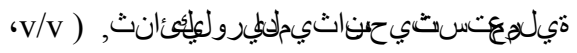

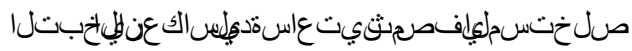

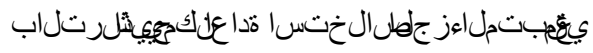
رب (Chang et al

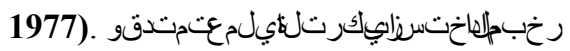

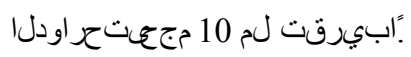

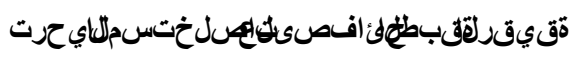
(TLC)

صلختسجل نغت لوركيام 00 اةبيمكتب حس

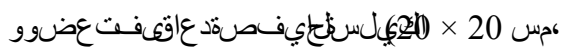

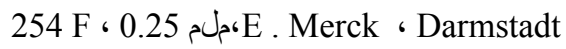
(Germany, حئافصطلياشنتمت نأقبثي

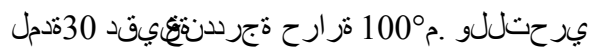

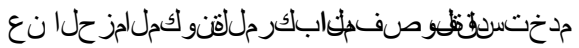

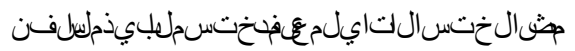
. قدسكأللةداضقليال|عفللهابتخ 1-

\section{Carotene)}

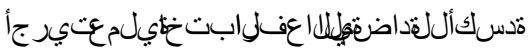

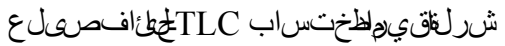

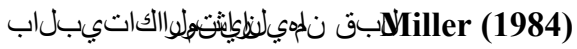

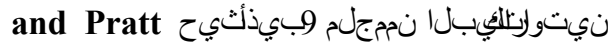

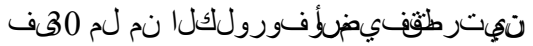

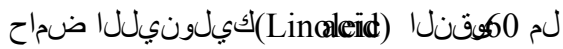

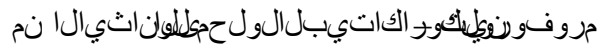

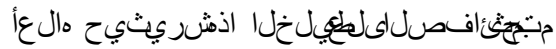

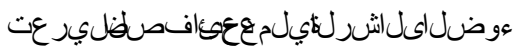

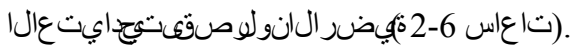

Arab Univ. J. Agric. Sci., 13(3), 2005 


$$
\text { دمحلاصوىدعسلا دمجهد عسل اليل علايخغب }
$$

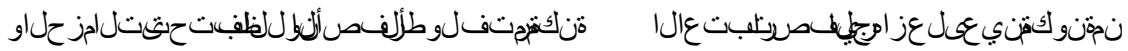

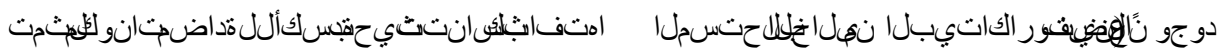

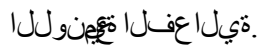

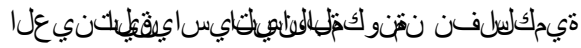

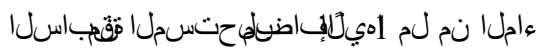

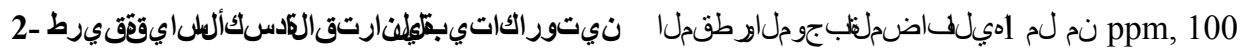

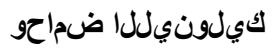

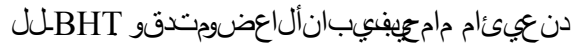

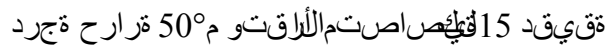

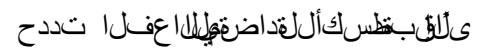

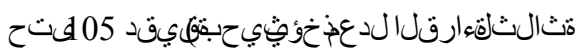

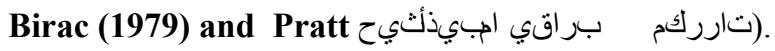

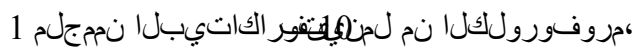

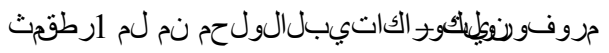

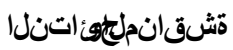

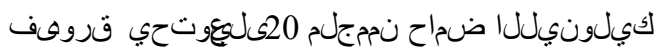

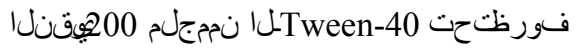

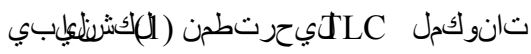

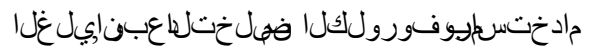

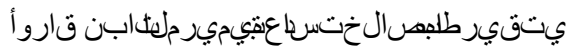

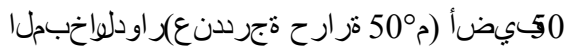

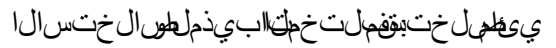

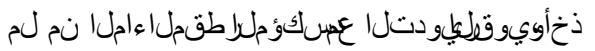

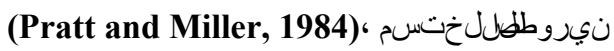
(Folch and ناسكثلاصلختسم ، (Sloanestanley,1957)

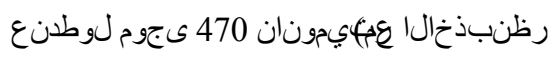




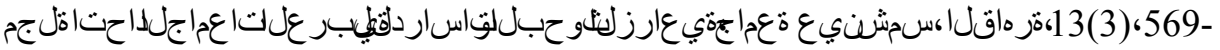

579، 2005

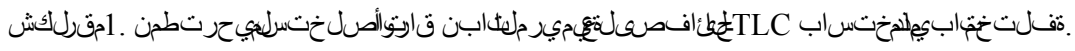

نيزنبلا -5 يدايتعالثاسكهل| -4

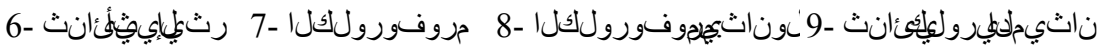

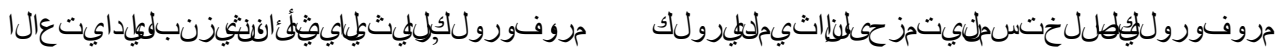

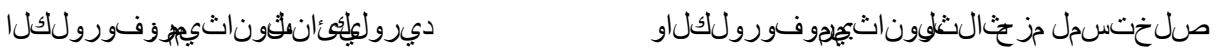

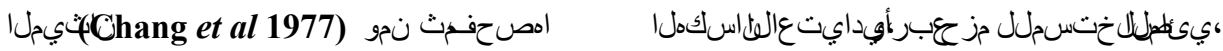

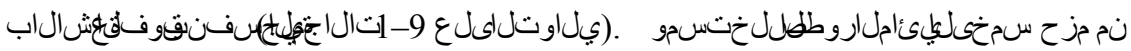

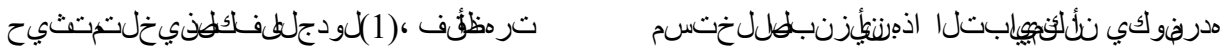

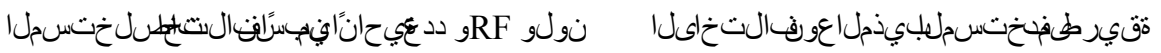

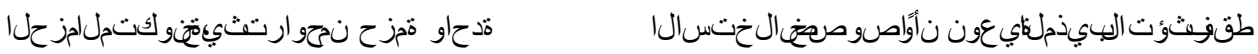

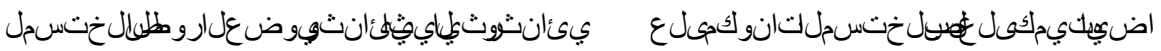

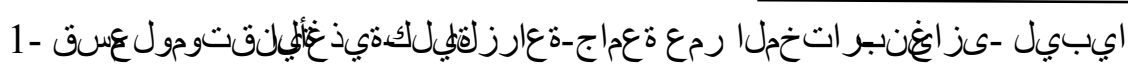

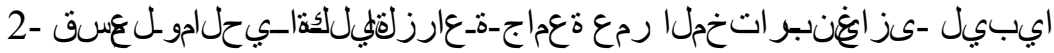


569

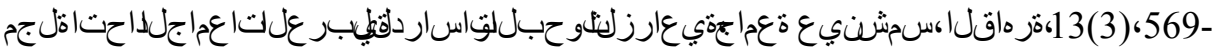
579، 2005

(Chang et al 1977 and Abd El-Aal \& Kalil, 1994).

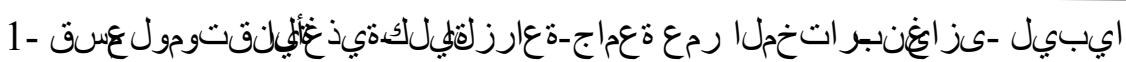

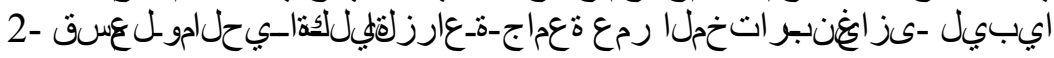

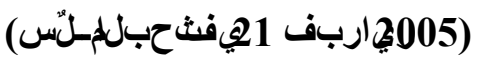

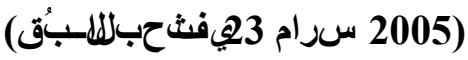




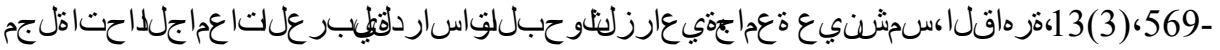
579، 2005

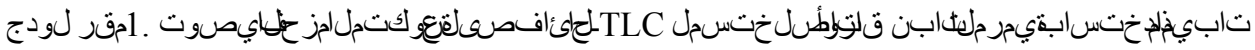

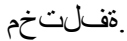

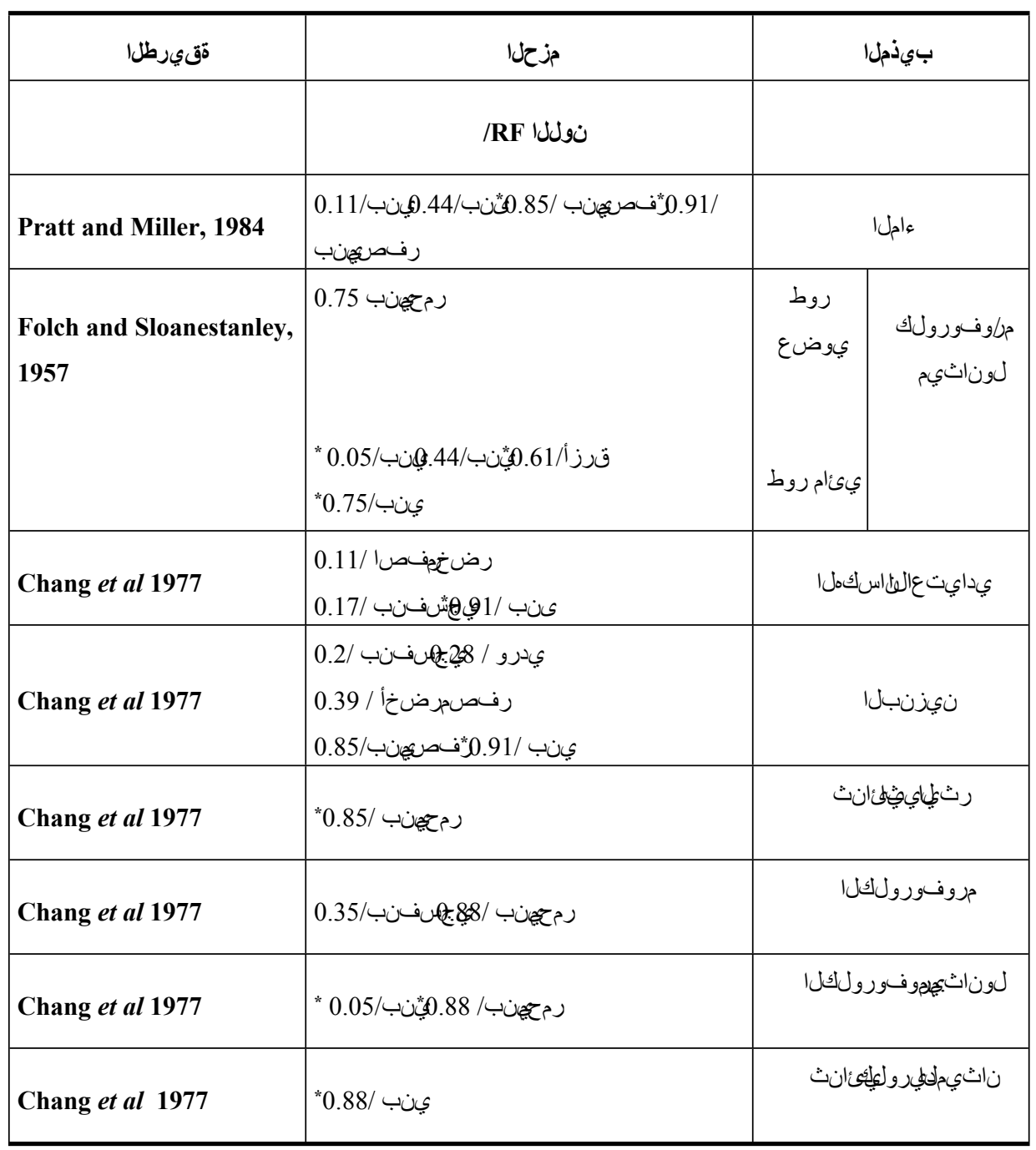

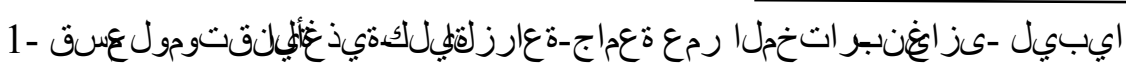

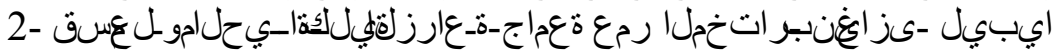

(2005)

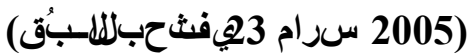


8

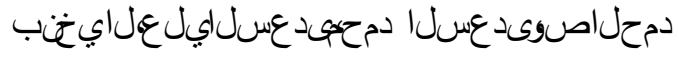

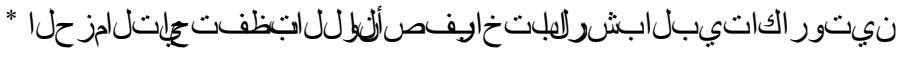




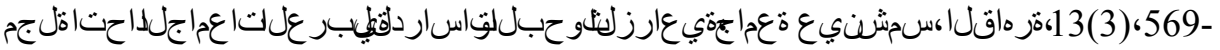
579، 2005

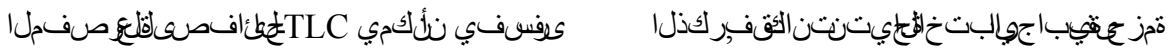

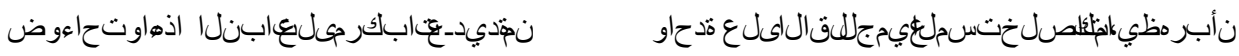

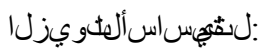

Borneol, Camphene, Camphor,

1,8-Cineol, $\alpha$ - Pinene and Thujone

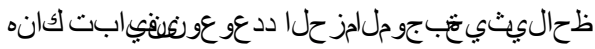

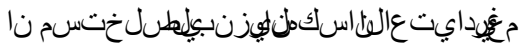

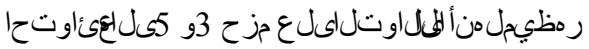

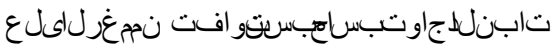
= 91 = دو جو نالعضف اذه (Svoboda, 1992) RF)، ثالثئئاملاروطلهل ققلثيلثالثابكرملا نمىر خألثلانوكملا نم ددع (BF) = 0.05, 0.61, 0.75) (Cuvelier et al 1994).

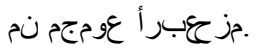

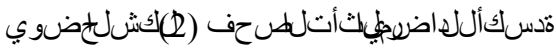

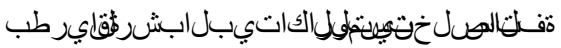

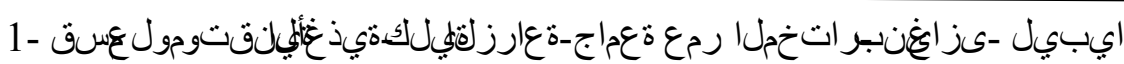

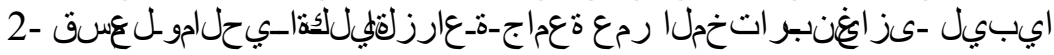




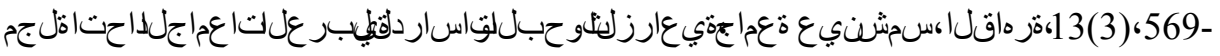
579، 2005

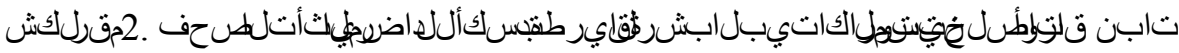

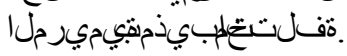

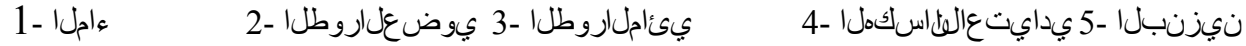

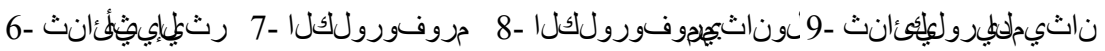

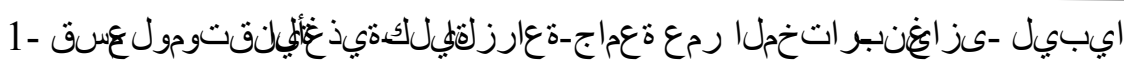

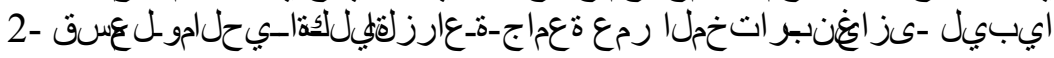




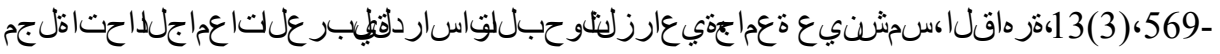
579، 2005

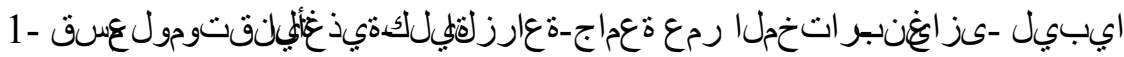

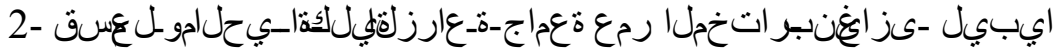


12

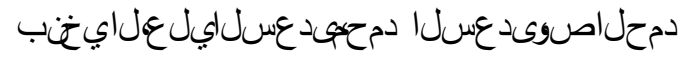

(Cuvelier et al 1994)

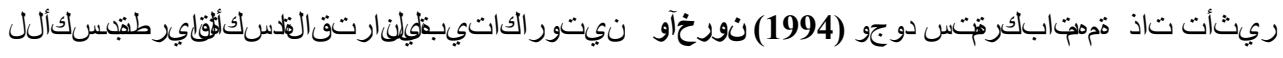

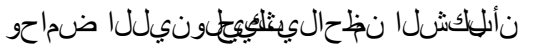

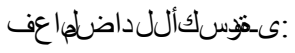

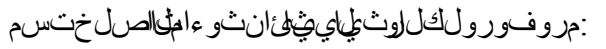

Carnosic acid, Carnosol, Epirosmanol,

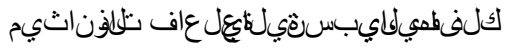

Methyl carnosat, Rosmad, Rosmanol

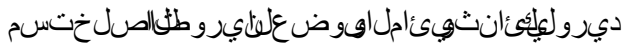

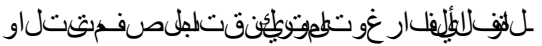

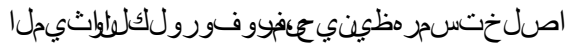

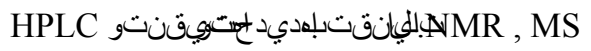

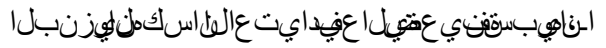

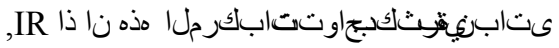

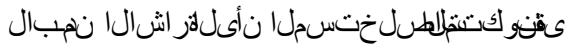

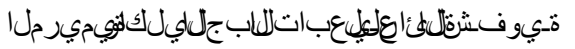

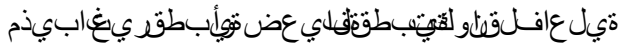

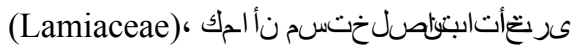

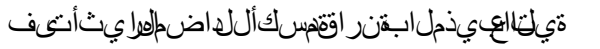

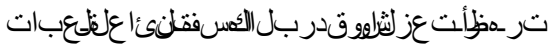

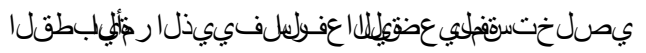

Chipault et

(Chang et al 1977).

al (1952) and Saito et al (1976)

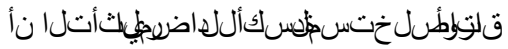

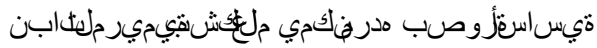

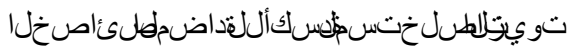

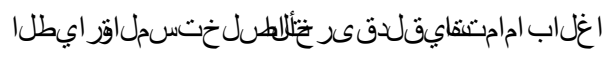

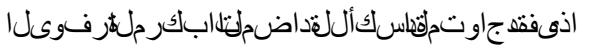

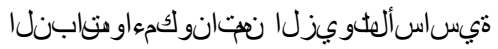

Bracco et al

(1981); Economou et al (1991) and

(Svoboda, 1992)

Gerhardt \& Schroter (1983) .

\section{REFERENCES}

Abd El-Aal, M.H. and M.K.M. Khalil

(1994). Faba bean hulls extract as a natural antioxidant and its effect on the stability of corn and sunflower oils. Com. in Sci. and Dev.Res . 677 (45) :119-138. Bracco, U.; J. Loliger and J.L. Viret (1981). Production and use of natural antioxidants. JAOCS . 58 : 686-690. Branen, A.L. (1975). Toxicology and biochemistry of BHA and BHT. $\boldsymbol{J} . \boldsymbol{A m}$.

Oil Chem . Soc . 52 : 59 -62.

Chang, S.S.; B. Ostric-Matijasevic; O.A.L. Hsieh and C. Huang (1977).
Natural antioxidants from rosemary and sage. J. Food Sci . 42 (4):1102-1106.

Chevolleau, S.; J.F. Mallet; E. Ucciani;

J. Gamisans and M. Gruber (1992).

Antioxidant activity in leaves of some

Mediterranean plants. JAOCS. 69 (12):

1269- 1271.

Chipault, J.R.; G.R. Mizuno; J.M.

Hawkins and W.O. Lundberg (1952).

The antioxidant properties of natural spices. Food Res. 17 : 46-50.

Cuvelier, M.E.; C. Berset and H.

Richard (1994). Antioxidant constituents in sage (Salvia officinalis). J. Agric.

Food Chem. 42: 665-669. 


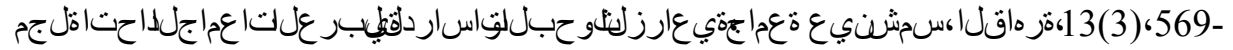
579، 2005

Duve, K.J. and P.J. White (1991).

Extraction and identification of antioxodants in oats. JAOCS . 68(6): 365-370.

Economou, K.D.; V. Oreopoulou and C.D. Thomopoulos (1991). Antioxidant activity of some plant extracts of the family Labiatae. JAOCS. 68 (2) : 109-112.

Eisa, E.S. (1999). Chemical study of natural antioxidant extracted from rice hulls. J. Agric. Sci . Mansoura Univ. 24 (2) : 847- 859 .

Folch, J.M. and G.H. Sloanestanley (1957). A simple method for isolation and purification of total lipids from animal tissues. J. Biol . Chem . 266- 497. Gerhardt, U. and A. Schroter (1983). Antioxidative effect of spices. Gordian, 9: $171-176$.

Jitoe, A.; T. Masuda; I.G.P. Tengah; D.N. Suprapta; I.W. Gera and N. Nakatani (1992). Antioxidant activity of tropical ginger extracts and analysis of the contained curcuminoids. J. Agric.

Food Chem. 40 (8): 1337-1342.

Johnson, A.R. and F.R. Hewgill (1961). The effect of the antioxidants, BHA, BHT and PG on growth, liver and serum lipids and serum sodium level of the rat. Australian Exp. Biol \& Med Sci. 39: 353-3357.

Kikuzaki, H. and N. Nakatani (1993). Antioxidant effects of some ginger constituents. J. Food Sci. 58(6):

1407-1410.

Kramer, R.E. (1985). Antioxidants in clove. JAOCS . 62 (1) : 111-113.
Lawrence, B.M. (1981). Essential oils, 1979-1980, p. 156. Allured Publishing Corporation, Wheaton, IL.

Miura, K. and N. Nakatani (1989). Antioxdative activity of biphenyl compounds from thyme (Thymus vulgaris L.). Chemistry Express, 4 (4): 237-239. Nakatani, N.; R. Inatani; H. Ohta and A. Nishioka (1986). Chemical constituents of peppers (Piper spp.)and application to food preservation: Naturally occuring antioxidative compounds. Environmental Health Perspectives, 67:135-138. Nakatani, N.; Y. Tachibana and $H$. Kikuzaki (1989). Antioxidative Compounds from Edible Plants: Phenolic amides from Capsicum frutescens L. p. 453. Hayashi, O.; E. Niki; M. Kondo and M. Yoshikawa (eds.), Elsevier science Publishers, B.V., Amesterdam.

Pratt, D.E. and P.M. Birac (1979). Source of antioxidant activity of soybeans and soy products. $\boldsymbol{J}$. Food $\mathbf{S c i}$. 44 : 1720-1724.

Pratt, D.E. and E.E. Miller (1984). A flavonoid antioxidant in Spanish peanuts. JAOCS . 61 (6): 1064 - 1071. Saito, Y.; Y. Kimura and T. Sakamoto (1976). The antioxidant effects of petroleum ether soluble and insoluble fractions from spices. Eiyo to Shokuryou, 29 (9): 505-508.

Svoboda, K.P. (1992). A study of the variability of rosemary and sage and their volatile oils on the British market: their

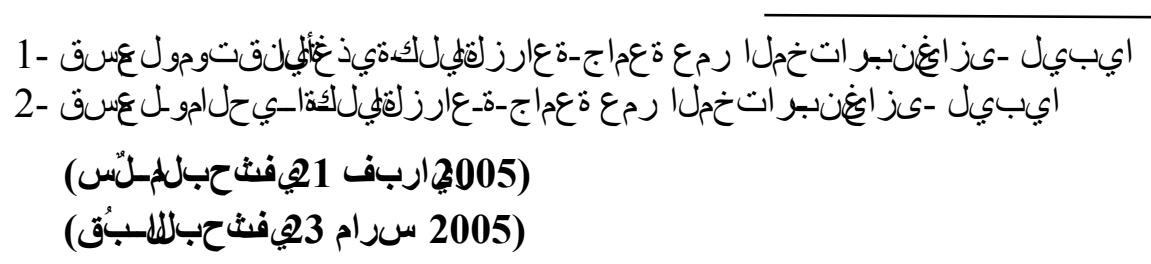


14

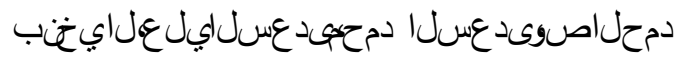

antioxidative properties. Flavour and

Fragrance J., 7:81-87.

cal structures of natural antioxidans from

Vekiari, S.A.; V. Oreopoulou; C. Tzia

rosemary. JAOCS. 59: 339-341.

and C.D. Thomopoulos (1993). Oregano

flavonoids as lipid antioxidants. JAOCS .

70 (5): 483-487.

Wu, J.W.; M.H. Lee; C.T. Ho and S.S.

Chang (1982). Elucidation of the chemi-

Univ. J. Agric. Sci., Ain Shams Univ., Cairo, 13(3), 569-579, 2005

\title{
ANTIOXIDANT ACTIVITY OF SOME SAGE LEAVE EXTRACTS
}

[38]

\author{
Benkhayal, F.A. ${ }^{2}$; A.H. Al-Saadi' ${ }^{1}$ M.H. Al-Saadi² and Wessal H. Hamad ${ }^{1}$
}

\begin{abstract}
Sage leaves were successively extracted with water, chloroform: methanol (2:1, $\mathrm{v} / \mathrm{v}), \mathrm{n}$ - hexane, benzene, diethyl ether, chloroform, chloroform: methanol $(8: 2, \mathrm{v} / \mathrm{v})$ and dichloromethan. Antioxidant activities were detected using two methods $: 1$. The carotene spray method and 2.Measuring the coupled oxidation of $\beta$-carotene and linoleic acid. Generally, all extracts had antioxidant activities when tested by the first method. By means of the second method, the extracts of water, diethyl ether and chloroform : methanol( $8: 2, \mathrm{v} / \mathrm{v})$ showed the greatest antixidant activities, followed by the extracts of organic and aqueous phases (which were extracted with chloroform: methanol, 2:1, v/v), dichloromethan and chloroform, while the nhexane and benzene extracts showed low activities.
\end{abstract}

Key words: Sage, Leaves, Extracts, Antioxidant, $\beta$ - Carotene.

1- Department of Food Science \& Technology, Faculty of Agriculture, University of Omar El-Mukhtar, Beni Ghazi, Libya.

2- Department of Biology, Faculty of Science, University of Omar El-Mukhtar, Beni Ghazi, Libya. 


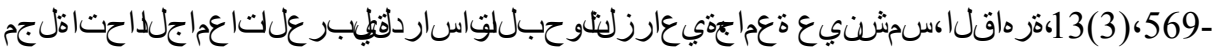
579، 2005

(Received February 21, 2005)

(Accepted March 23, 2005)

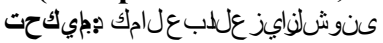

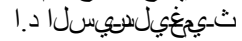

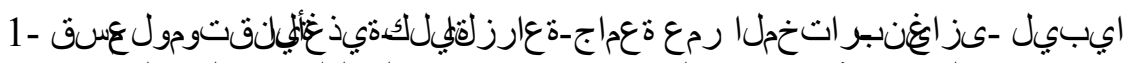

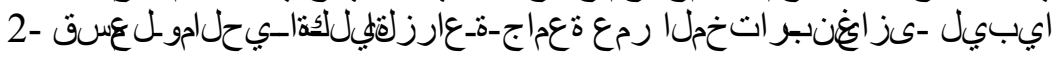

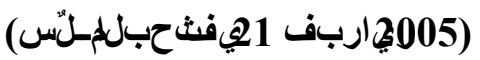

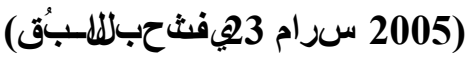

